\title{
SYNTHESIS AND CHARATERIZATION OF COPPER NANOPARTICLES AND EVALUATION OF ANTIBACTERIAL ACTIVITY
}

\author{
N. Karikalan* \\ ${ }^{1}$ Assistant Professor Senior Scale, Department of Physics, Hindustan Institute of Technology and \\ Science, Chennai, Tamil Nadu, India \\ *E-mail : sivakanikari@gmail.com
}

\begin{abstract}
The nanotechnology has been developed in recent years due to the wide application in the human beings. One of the famous nanomaterials is copper nanoparticles. The copper nanoparticles have been reported to have strong antibacterial activity. Nowadays there are many environmental problems and illness caused by the microbial pathogen. It is widely agreed that nanoparticles are clusters of atoms in the size range of 1-100 nanometer. The present study included the chemical reduction of copper chloride through trisodium citrate and testing for their antimicrobial activity. The aqueous copper chloride exposed to the trisodium citrate results in the synthesis of copper nanoparticles. The $\mathrm{pH}$ and viscosity of the copper nanoparticles monitored and indicates the stability of the particles. Ultraviolet-visible spectrum and Fourier-transform infrared spectroscopy further confirmed in the synthesized copper nanoparticles. These synthesized copper nanoparticles were further confirmed by using a scanning electron microscope. The scanning electron microscope analysis showed the particle size between 44.50 nanometers as well the spherical structure of the nanoparticles. The proven antibacterial activity of synthesized copper nanoparticles against Escherichia coli established. It is confirmed that copper nanoparticles are capable of rendering high antibacterial efficacy and hence has a great potential in the preparation of drugs used against bacterial diseases. Applications of copper nanoparticles based on these findings may lead to valuable discoveries in various fields such as medical devices and antimicrobial systems.
\end{abstract}

Keywords: Nanotechnology, copper nanoparticles, Trisodium citrate

(c) RASĀYAN. All rights reserved

\section{INTRODUCTION}

The nanotechnology has been developed in recent years due to the wide application in the human beings. One of the famous nanomaterials is copper nanoparticles. The Cg-np has been reported to have strong antibacterial activity. Nowadays there are many environmental problems and illness caused by the microbial pathogen ${ }^{1-2}$. Nanotechnology is the arts and science of manipulating matter at the atomic or molecular scale and holds the promise of providing significant improvements in technologies for protecting the environment. While many definitions for nanotechnology exist, the United States Environmental Protection Agency uses the definition developed by the National Nanotechnology Initiative. According to National Nanotechnology Initiative of the United States of America, nanotechnology is defined as research and technology development at the atomic, molecular or macromolecular levels using a length scale of approximately one to one hundred $\mathrm{nm}$ in any dimension the creation and use of structures, devices and systems that have novel properties and functions because of their small size and the ability to control or manipulate matter on an atomic scale ${ }^{3}$. The technology has excellent prospects for exploitation across the medical, pharmaceutical, biotechnology, engineering, manufacturing, telecommunications and information technology markets. Copper nanoparticles, due to their excellent physical and chemical properties and low cost of preparation have been of great interest. Copper nanoparticles have wide applications as heat transfer systems, antimicrobial materials, super strong materials, sensors and catalysts. Copper nanoparticles are very reactive because of their high

Rasayan J. Chem., 11(4), 1451-1457(2018) http://dx.doi.org/10.31788/RJC.2018.1143068

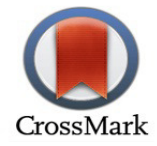


surface-to-volume ratio and can easily interact with other particles. In the present study the synthesis, characterization and application of copper nanoparticles using Tri sodium citrate as reducing agent.

\section{EXPERIMENTAL}

Materials and Methods

Copper chloride $\left(\mathrm{CuCl}_{2}\right)$ and Tri sodium citrate $\left(\mathrm{Na}_{3} \mathrm{C}_{6} \mathrm{H}_{5} \mathrm{O}_{7}\right)$ Loba Chemi, India have been used in the synthesis of Copper Nanoparticles.

\section{Preparation of Copper Nanostructures}

Copper nanoparticles by sodium citrate method. Copper chloride $\left(\mathrm{CuCl}_{2}\right)$ solution $(5$ gram in 100 milliliter water) was prepared first and to it was added drop-wise a solution of sodium citrate so as to maintain a ratio of 1:2 or 1:5. After about $30 \mathrm{~min}$, an aq. a solution of sodium formaldehyde sulfoxylate (SFS) (1:1 with respect to $\mathrm{CuCl}_{2}$ ) was added to the reaction mixture with rapid stirring at room temperature and the reaction mixture was further stirred for an hour below $60^{\circ} \mathrm{C}$ to obtain a brown precipitate. The powder was collected by centrifugation followed by washing and was dried in a vacuum oven for 2-3 hours.

\section{Characterization of Copper Chloride Nanoparticles Measurement of pH}

The $\mathrm{pH}$ of the solution was monitor using a digital $\mathrm{pH}$ meter. The measurement was taken until the synthesized copper nanoparticles.

\section{Ultraviolet-Visible Analysis}

The extracts were examined under visible Ultraviolet-visible spectrum. The sample is dissolved in the same solvent. The extracts were scanned in the wavelength ranging from 340-960 nanometer using Systronic Spectrophotometer. These solutions were scanned in turn at intervals of $10 \mathrm{~nm}$ and the characteristic peaks were detected. The peak value of the UV-Visible was recorded.

\section{Fourier Transform Infrared Spectroscopy}

To determine Fourier transform infra-red (FTIR) pattern of the copper sulphate nanoparticles was freezedried and the dried powder was diluted with potassium bromide in the ratio of 1:100 and recorded the spectrum in Perkin Elmer FTIR Spectrum BX (Wellesley, MA, USA).

\section{SEM Analysis of Copper Nanoparticles}

The scanning electron microscopy (SEM) analysis of the freeze-dried sample was performed by mounting nanoparticles on specimen stubs with double-sided adhesive tape and coated with platinum in a sputter coater and examined under VEGA3 SEM (Japan) at $10 \mathrm{kV}$.

\section{Determination of Viscosity}

The viscosity of the crystal solution (100 milligrams in 100 milliliters of distilled water) was determined by using Oswald's viscometer. A volume of water usually 10 to $25 \mathrm{ml}$ depending upon the capacity of the bulb, pipetted into the lower mark. It is then sucked into the upper mark with the help of the rubber tube. In the end, it rises to the upper mark. The time taken by the water to flow through the capillary tube lower to upper mark is noted by means of a stopwatch. Let it be t1. The viscometer is dried and the same volume of the liquid under examination is taken and the process is repeated as before. Let the time of flow be $\mathrm{t} 2$. Then,

$$
\eta 1 / \eta 2=\mathrm{d} 1 \mathrm{t} 1 / \mathrm{d} 2 \mathrm{t} 2
$$

Where, $\mathrm{d} 1$ and $\mathrm{d} 2$ are the densities of the two liquids and $\eta 1$, and $\eta 2$ is the coefficient of viscosities of the two liquids (one of which is water). The viscosity of the liquid can be easily calculated.

\section{Determination of Antimicrobial Activity}

Antibiogram was done by disc diffusion method ${ }^{4-5}$ using samples. Petri plates were prepared by pouring $30 \mathrm{ml}$ of Nutrient agar medium for bacteria. The test organism was inoculated on a solidified agar plate 
with the help of micropipette and spread and allowed to dry for 10 mints. The surfaces of media were inoculated with bacteria from the culture. A sterile cotton swab is dipped into a standardized bacterial test suspension and used to evenly inoculate the entire surface of the Nutrient agar plate. Briefly, inoculums containing bacteria species were spread on Nutrient agar plates. Using sterile forceps, the sterile filter papers (6 millimeter diameter) containing the crude sample $(50 \mu \mathrm{l}, 100 \mu \mathrm{l}, 150 \mu \mathrm{l})$ were laid down on the surface of the inoculated agar plate. The plates were incubated at $37^{\circ} \mathrm{C}$ for $24 \mathrm{~h}$ for the bacteria. Each sample was tested in triplicate. The antimicrobial potential of test compounds was determined on the basis of the mean diameter of the zone of inhibition around the disc in millimeters. The zones of inhibition of the tested microorganisms by the samples were measured using a millimeter scale.

\section{RESULTS AND DISCUSSION}

Production of nanoparticles can be achieved through different methods. Chemical approaches are the most popular methods for the production of nanoparticles. Copper nanoparticles, due to their excellent physical and chemical properties and low cost of preparation, have been of great interest. Copper nanoparticles have wide applications as heat transfer systems, antimicrobial materials, super strong materials, sensors and catalysts. Copper nanoparticles are very reactive because of their high surface-tovolume ratio and can easily interact with other particles and increase their antimicrobial efficiency. In the present study to synthesize, characterization and antibacterial activity of copper nanoparticle using trisodium citrate.

\section{Synthesis of Copper Nanoparticles}

The synthesis of Copper chloride nanoparticles through trisodium citrate was carried out. Copper is used as a reducing agent as Copper chloride has distinctive properties such as good conductivity, catalytic and chemical stability. Applications of such eco-friendly nanoparticles in bactericidal, wound healing and other medical and electronic applications, makes this method potentially exciting for the large-scale synthesis of other inorganic materials (nanomaterials). Copper chloride and trisodium citrate were used as starting materials for the preparation of copper nanoparticles. The copper colloid was prepared by using chemical reduction method ${ }^{6}$. The mechanism of reaction could be expressed as follows ${ }^{7-8}$.

The aqueous Copper, when exposed to trisodium citrate, was reduced in solution, there by leading to the formation of Copper chloride hydrosol. The time duration of the change in color varies from chemical to chemical. It is well known that Copper nanoparticles exhibit in aqueous solution due to excitation of surface plasmon vibrations in nanoparticles ${ }^{9}$.

\section{pH and Viscosity of Copper Nanoparticles}

The $\mathrm{pH}$ value of Copper nanoparticles is 3.56 (Table-1). The $\mathrm{pH}$ of the Copper nanoparticles was in accordance with the USP guidelines for topical and transdermal formulations. The $\mathrm{pH}$ of the CuNPs is 3.56. It is the acidic condition. Due to the presence of higher no of $\mathrm{H}^{+}$and lower $\mathrm{OH}^{-}$ions in the sols with pH 3.56 is observed.

In the case of very high solubility (i.e. solutions containing a large number of solute) rate may be very low due to the increased solution viscosity which renders the system diffusion controlled or the resulting nanoparticles. Nanoparticle size is the dependence of the viscosity of the surrounding medium. In the present study, the viscosity of the CuNPs was 0.985 (Table-1). The increased viscosity nanoparticle support the stabilization of nanoparticles.

\section{Ultraviolet/Visible (UV/VIS) Spectroscopy}

The Copper nanoparticles show a good transmittance in the visible region which enables it to be a good material for optoelectronic applications. Spectroscopy is a tool for structure determination. It is essentially a technical procedure by which the energy differences between the allowed states of a system are measured by determining the frequencies of the corresponding light absorbed. The obtained spectra of the compound i.e. the response of a substance subjected to radiations of various wavelengths reveals the important properties of the compound. 
UV-Vis- spectroscopy is used to measure the absorption or emission of radiation associated with the spatial distribution of electrons in atoms or molecules. For this purpose, transmission is plotted against wavelength or frequency and is known as the transmission spectrum. The UV-Visible- transmission spectra were recorded using Systronic UV-Visible Spectrometer in the range $340 \mathrm{~nm}$ to $940 \mathrm{~nm}$. Figure-1 shows the transmission spectra Copper Nanoparticles. From the spectra, it is observed that the show Copper nanoparticles good transmittance in the entire visible - regions.

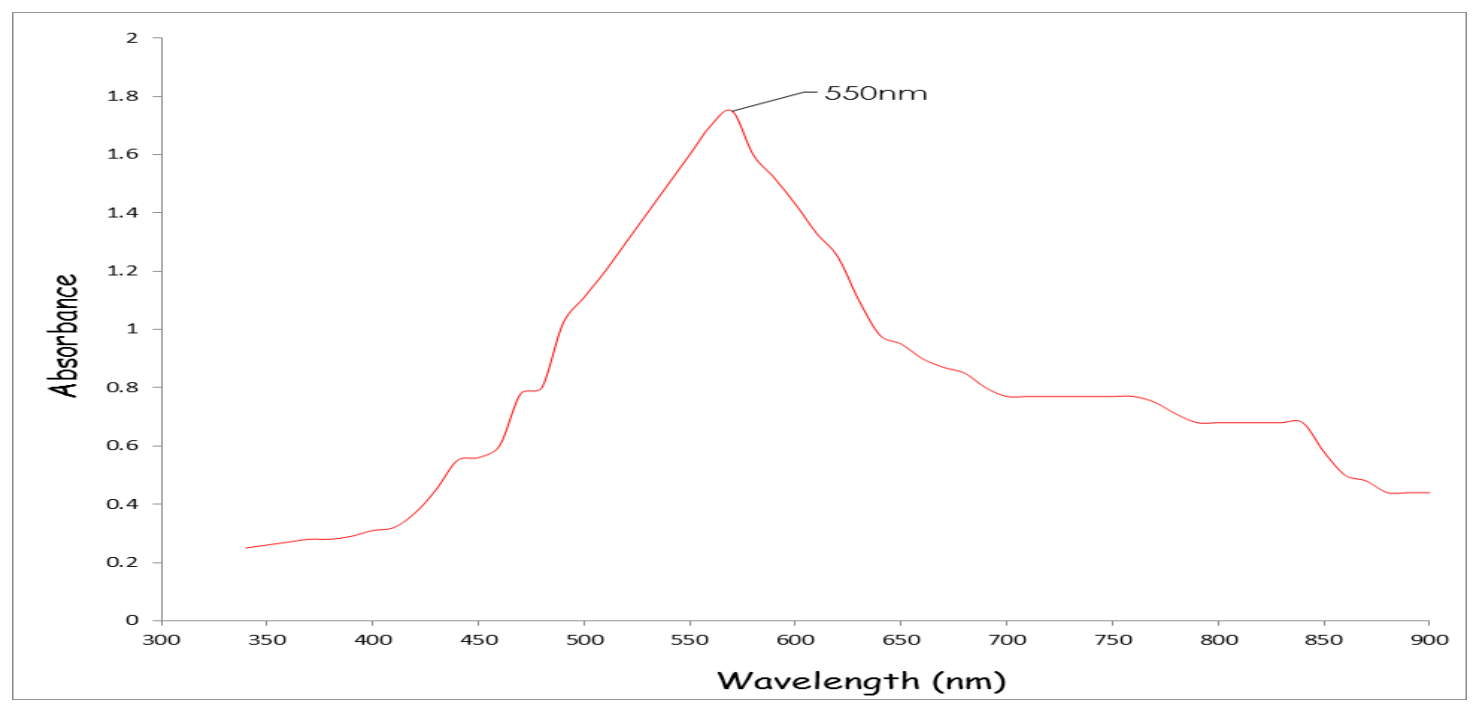

Fig.-1: Shows the UV Visible Spectrum of CuNP

The absorption obtained spectra were compared with the absorption spectra of copper nanoparticles prepared using these extracts in order to reveal the formation of copper phyto-nanoparticles. The absorption spectra of copper phytonanoparticles were recorded after 24 hours after their preparation and exhibited absorbance peaks at $550 \mathrm{~nm}$.

Fourier Transform Infrared Spectroscopy (FTIR) Analysis FTIR is an important tool which enables us to understand the involvement of functional groups in the interactions between metal particles and biomolecules. Fourier Transform Infrared (FTIR) spectroscopy can effectively be used to measure the particle formation. It is found that the width and intensity of peaks in an IR spectrum have explicit dependence on the particle size. As particle size increases, the width of the peak decreases and intensity increases ${ }^{10}$. The FTIR spectra of reduced Copper chloride show in the peaks of spectra in Fig.-2.

The band of carboxyl or carbonyl groups at 1660 to 1500 and 1390 to $1260 \mathrm{~cm}^{-1}$ region. This may be the reason for the reduction of the transmittance at this region in the case of the spectrum of nanoparticles. The shift of the band from 1656 to 1586 indicates the formation of metal carbonyl groups. It is due to the stabilization of $\mathrm{Cu}$ nanoparticles by the -COO- a group of trisodium citrate. This asymmetric shift can be comparable with the data presented by previous works ${ }^{11}$. According to them, when the citrate ligand bound to magnetite nanoparticles surfaces the antisymmetric stretching of -COO- at $1586 \mathrm{~cm}^{-1}$ almost remains the same but the symmetric -COO- stretching mode of citrate becomes redshifted and appears sharply ${ }^{12}$ at $1398 \mathrm{~cm}^{-1}$.

Table-1: $\mathrm{pH}$ and Viscosity of Copper Nanoparticles

\begin{tabular}{c|c|c}
\hline S. No. & Parameters & Copper Nanoparticles \\
\hline 1 & $\mathrm{pH}$ & 3.56 \\
\hline 2 & Viscosity (Pos) & 0.985 \\
\hline
\end{tabular}




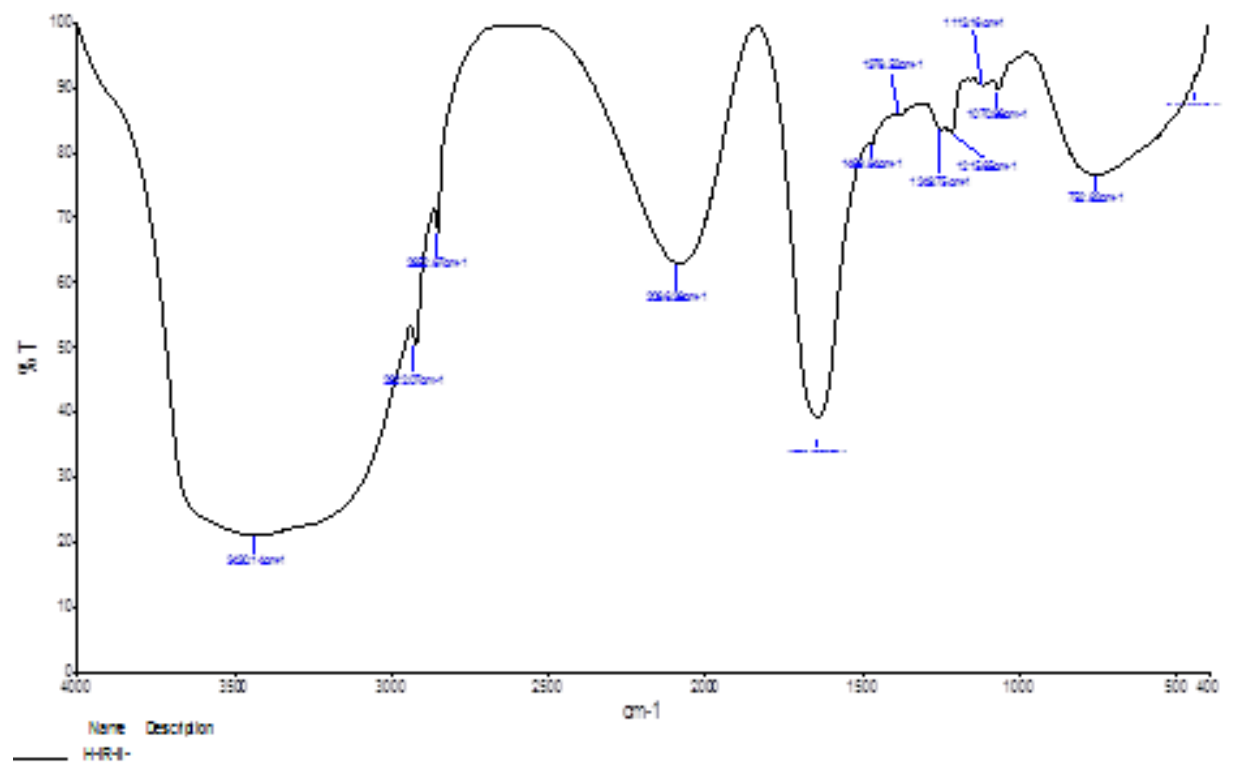

Fig.-2: FTIR Analysis of Copper Nanoparticles

\section{SEM Analysis}

SEM analysis was carried out to understand the topology and the size of the Cu-NPs, which showed the synthesis of higher density polydispersed spherical $\mathrm{Cu}$-NPs of various sizes. The SEM image showing the high-density copper nanoparticles synthesized by trisodium citrate further confirmed the development of copper nanostructures. Most of the nanoparticles aggregated and only a few of them were scattered, as observed under SEM. The SEM analysis showed the particle size $44.50 \mathrm{~nm}$ as well the spherical structure of the nanoparticles (Fig.-3).

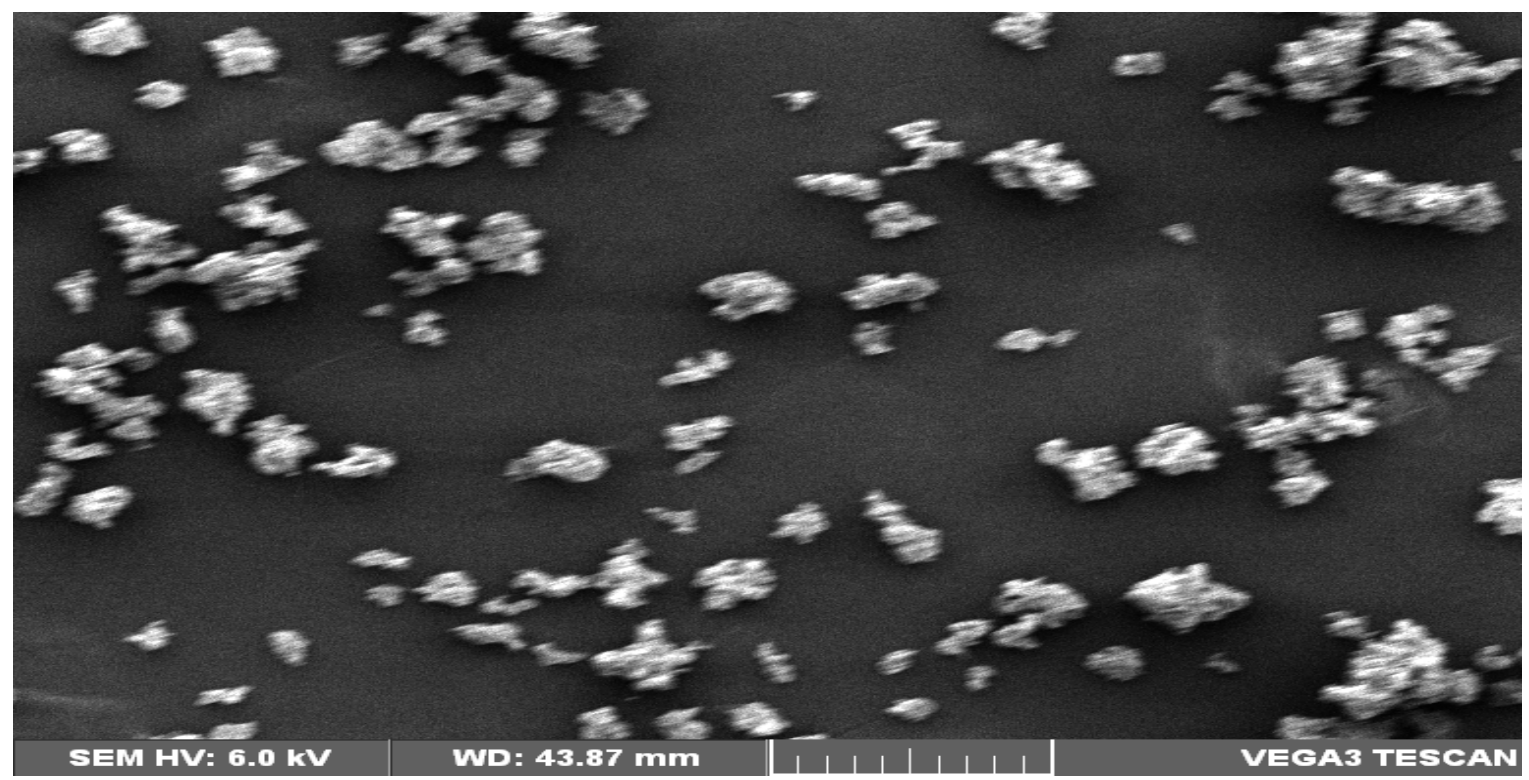

Fig.-3: High-Resolution Scanning Electron Microscopic (SEM) Image of the Copper Nanoparticles (CuNPs). Spherical shaped CuNPs size at $44.50 \mathrm{~nm}$

\section{Antibacterial Activity of Copper Nanoparticles}

Microbes are truly the most underappreciated living organisms on Planet Earth. Billions of them can fit on a fingernail, and they make up more than half of the living biomass on the planet. The world we live in is one full of microbes. Microbes, whether they are good, bad, or benign, are certainly everywhere. This 
includes on our body, in our homes, far below the earth's surface and up to the atmosphere, in cold, cool, warm and hot and very hot places, and even in places without oxygen. Our body temperature and wealth of nutrients provide an ideal home for these micro-organisms to thrive. Microorganisms always live in water (directly in aquatic environments, in water inside animals or plants, or in the water around soil particles). They can eat all sorts of things, including oil, rocks, dead and living plants and animals ${ }^{13}$. There are 4 major types of Microbes: bacteria, fungi, protists and viruses. ${ }^{14}$

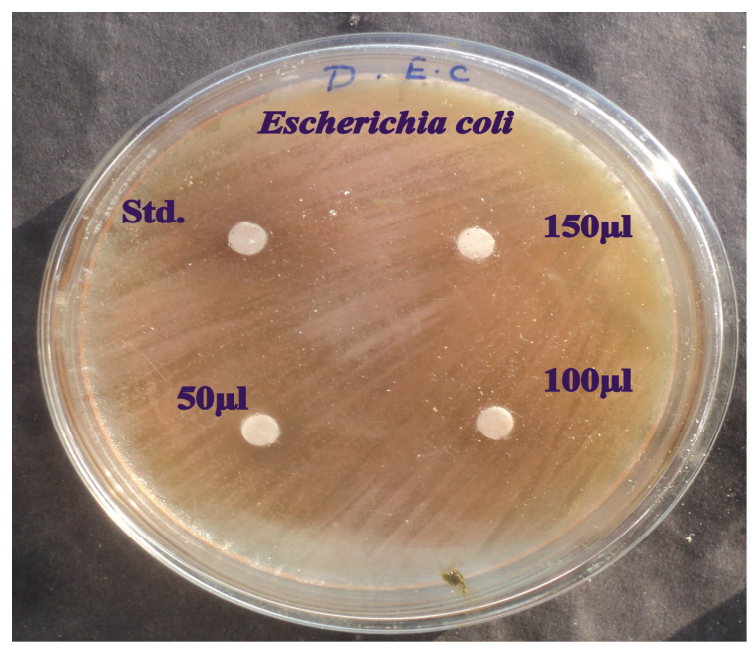

Fig.-4: Antibacterial Activity of Copper Nanoparticle

The in the vitro antimicrobial activity of the Copper nanoparticles against these bacteria was qualitatively assessed by the presence of inhibition zones represented in the photographic Fig.-4. The inhibitory activities in culture media of the Copper nanoparticles reported in Table-2 were comparable with standard antimicrobiotic viz. Chloramphenicol.

\section{CONCLUSION}

The present study exhibit a simple method of synthesis of copper nanoparticles from a novel primitive chemical source. This method can be further used for industrial production of nanoparticles at room temperature and with a single step. Since the nanoparticles thus synthesized shows antimicrobial activity, they can be used in the field of pharmaceutical industry. Copper nanoparticles might be useful for the development of newer and more potent antimicrobial agents. All the above data's represented in our study contribute to a novel and unexplored area of nanomaterials as medicine.

\begin{tabular}{c|c|c|c|c}
\multicolumn{5}{c}{ Table-2: Antibacterial Activity of Copper Nanoparticles } \\
\hline Microbe & $50 \mu 1$ & $100 \mu 1$ & $150 \mu 1$ & Standard \\
\hline $\begin{array}{c}\text { Escherichia coli } \\
(\mathrm{mm})\end{array}$ & $5.23 \pm 0.36$ & $7.50 \pm 0.52$ & $8.75 \pm 0.61$ & $9.25 \pm 0.64$ \\
\hline \multicolumn{5}{c}{ Values are expressed Mean \pm SD for triplicates }
\end{tabular}

\section{ACKNOWLEDGMENT}

The authors would like to thank the Hindustan Institute of Technology and Science for their support.

\section{REFERENCES}

1. E. S. Nour, C. O. Chey, M. Willander, and O. Nur, Nanotechnology, 26, 095502(2015), DOI: $10.1088 / 0957-4484 / 26 / 9 / 095502$.

2. Sudhindra E. Sant, Nanoparticles: From Theory to Applications Günter Schmid, 2nd ed., Wiley-VCH Verlag, Weinheim, 2010, pp.1462-1463, DOI: 10.1080/10426914.2012.663137.

3. P. R. Somani, S. P. Somani, E. Flahaut, and M. Umeno, Nanotechnology,18,185708(2007), DOI: $10.1088 / 0957-4484 / 18 / 18 / 185708$ 
4. NCCLS. National Committee for Clinical Laboratory Standards. Performance standards for antimicrobial disc susceptibility tests. PA: NCCLS Publications, 25, (1993), DOI: 10.1093/jac/dkf093.

5. O.A. Awoyinka, I.O. Balogun, and A.A. Ogunnow, J. Med. Plant Res., 1, 63(2007), DOI: 10.5897/JMPR/2007.05.18.

6. P. Mohanpuria, N. K. Rana and S.Yadav, J. Nanopart. Res., 10(3), 507(2010), DOI: 10.1007/s11051007-9275-X

7. E. M. Silva, J. N. S. Souza, H. Rogez, J. F. Rees and Y. Larondelle, Food Chemistry, 101, 1012 (2007), DOI:10.1016/j.foodchem.2006.02.055.

8. X. Hangxun and L. Kenneth, Nanoscience and Nanotechnology, An International Journal, 5(2), 9 (2010), DOI: 10.1615/NanomechanicsSciTechnolIntJ.v1.i2

9. A. K. Mamun, Y. Chisti and U. C. Banerjee, Biotechnology Advances, 31(2), 346(2012), DOI: 10.1016/j.jsps.2012.11.013.

10. I. Thomann, B. A. Pinaud, Z. Chen, B. M. Clemens, T. F. Jaramillo and M. L. Brongersma, Nano Lett.,11, 3440 (2011), DOI:10.1021/nl201908s.

11. Y. Zi1 and Z. L.Wang, APL Materials, 5, 074103 (2017), DOI: 10.1063/1.4977208.

12. Garima Pandey, Rasayan J. Chem., 11(3), 942(2018), DOI:10.31788/RJC.2018.1133031

13. E. S. Nour, C. O. Chey, M. Willander, and O. Nur, Nanotechnology, 26, 095502 (2015), DOI:10.1088/0957-4484/26/9/095502.

14. I. Linkov, Nanomaterials, New York, Springer (2008), pp.58-62.

[RJC-3068/2018] 